\title{
ac-field-induced fluid pumping in microsystems with asymmetric temperature gradients
}

\author{
Moritz Holtappels, ${ }^{1}$ Marco Stubbe, ${ }^{2}$ and Jan Gimsa ${ }^{2, *}$ \\ ${ }^{1}$ Max Planck Institute for Marine Microbiology, Celsiusstrasse 1, D-28359 Bremen, Germany \\ ${ }^{2}$ Chair of Biophysics, Faculty of Biology, University of Rostock, D-18057 Rostock, Germany
}

(Received 27 May 2008; revised manuscript received 4 December 2008; published 12 February 2009)

\begin{abstract}
We present two different designs of electrohydrodynamic micropumps for microfluidic systems. The micropumps have no movable parts, and their simple design allows for fabrication by microsystems technology. The pumps are operated by ac voltages from 1 to $60 \mathrm{~V}$ and were tested with aqueous solutions in the conductivity range of $1-112 \mathrm{mS} \mathrm{m}^{-1}$. The pump effect is induced by an ac electric field across a fluid medium with an inhomogeneous temperature distribution. It is constant over a wide range of the ac field frequency with a conductivity-dependent drop-off at high frequencies. The temperature-dependent conductivity and permittivity distributions in the fluid induce space charges that interact with the electric field and induce fluid motion. The temperature distribution can be generated either by Joule heating in the medium or by external heating. We present experimental results obtained with two prototypes featuring Joule heating and external heating by a heating filament. Experimental and numerical results are compared with an analytical model.
\end{abstract}

DOI: 10.1103/PhysRevE.79.026309

\section{INTRODUCTION}

The trend towards miniaturization in the fields of chemistry, environmental monitoring, medical diagnostics, bioengineering, and cell biology has brought an increasing number of integrated microdevices for chemical and biological applications. These are known as chemical micro-total-analysis systems ( $\mu$ TAS) or biological lab-on-a-chip systems [1,2]. The latter are used for cell monitoring [3-5] and cell sorting [6] or as microbioreactors $[1,2,7,8]$. A challenging problem in all such systems is the precise handling of liquid microvolumes [9], which requires self-contained micropumps of small package size exhibiting either a very small displacement volume (displacement pumps [10]) or a continuous volume flow (dynamic pumps [10]). For clarity, we here designate the latter class of pumps as dynamic field pumps. Different dynamic field pumping principles have been developed utilizing the interactions of the fluid with an electric and/or magnetic field. They are based on magnetohydrodynamic [11,12], electro-osmotic [13-15], or electrohydrodynamic effects [16-19]. The main advantage of dynamic field pumps is the lack of moving mechanical components. This prevents abrasion and simplifies microfabrication. In contrast to displacement pumps, the performance of dynamic field pumps benefits from downsizing because electric fields of high magnitude are more easily generated over small scales and heat dissipation is facilitated by the increased surface-tovolume ratio.

Dynamic field pumps exploit the interaction of the electric field with the medium. In electro-osmotic micropumps, fluid flow is generated by the electric force on charges in the diffusive double layer at interfaces such as exist between the aqueous solution and the electrodes or other solid surfaces. Static and dynamic electric fields can be used that are generated by two electrodes $[14,15]$, arrays of asymmetrically shaped pairs of electrodes, or arrays of traveling-wave elec-

\footnotetext{
*Corresponding author. FAX: +49-381-498 6022. jan.gimsa@uni-rostock.de
}

PACS number(s): 47.61.Fg, 47.10.-g, 47.65.-d, 85.90.+h

trodes operated in the $\mathrm{Hz}$ and low-kHz ranges [13,20-22]. Electro-osmotic pumping is a clear surface effect; hence, it is preferentially applied in fluids of low ion concentrationi.e., low conductivity-since the characteristic thickness of the diffusive double layer (Debye-Hückel length) inversely depends on the ion concentration in the fluid. A lower conductivity results in a driving force induced at a larger distance from the interface, thus reducing the viscous drag. Furthermore, Joule heating in the volume is reduced at lower medium conductivities.

Traveling waves in the $\mathrm{kHz}$ and $\mathrm{MHz}$ ranges can be used to propel dielectric elements or to pump fluids [23,24]. Traveling-wave micropumps have been described in detail by Fuhr et al. [18]. In these pumps, traveling waves of an electric field along an electrode array interact with space charges in the volume of the medium. The induction of space charges requires conductivity and/or permittivity gradients in the fluid, which can be induced by a temperature gradient. The pump effect is most efficient at a characteristic distance from the electrode array corresponding to the electrode dimensions. The effect is generated by the out-of-phase part (mathematically, the imaginary part) of the induced medium polarization $[23,24]$. Accordingly, the traveling-wave force is a peak function of the field frequency, similar to the Lorentzian torque peaks in electrorotation. The force reaches a maximum for $\omega \tau=1$, where $\omega$ is the angular frequency of the electric field and $\tau$ is the charge relaxation time of the fluid.

Green et al. [25] observed electrothermally induced fluid flow in the vicinity of a pair of symmetric microelectrodes located in a symmetric channel. The applied ac field heats the fluid and interacts with free space charges that are induced due to the temperature-dependent conductivity and permittivity gradients in the fluid. Nevertheless, the net flow is zero since the circular convections at the two electrodes are oppositely directed.

In this paper, we demonstrate that a fluid net flow is induced in a biased temperature field. We compare two different types of dynamic field micropumps and present an analytical description. In both pump types, the ac field is generated by two electrodes in the pumping medium, which exhibits a temperature gradient. In the first type, this gradient 
is generated by the inhomogeneous field distribution in a funnel-shaped pump channel that causes an inhomogeneous distribution of Joule (i.e., Ohmic) heating [26]. In the second type, recently described by us $[27,28]$, the temperature gradient is generated by the asymmetric location of an electric heating element in a homogenous pump channel.

The application of the micropumps is not restricted to low conductivities and shows constant pumping velocities in a wide frequency range of the electric field. Unlike the traveling-wave pump, the force effect is related to the inphase part (mathematically, the real part) of the polarization in the medium; i.e., the effect is comparable to a dielectrophoretic medium translation.

\section{THEORY}

\section{A. Electrohydrodynamic forces based on structural (Maxwell-Wagner) polarization}

The dielectrophoretic displacement of particles can be influenced by field-induced fluid convections. This distorting effect has motivated the detailed discussion of the interaction of the electric field with the fluid [29]. In a gedankenexperiment, the electrohydrodynamic force can be considered a dielectrophoretic force on a "fluidic particle with thermally induced smeared interfaces." The time-averaged force on an infinitesimally small volume element of a fluid exposed to an electric field varying over time is [29]

$$
\left\langle F_{E}\right\rangle=-\frac{1}{2}\left[\left(\frac{\nabla \sigma}{\sigma}-\frac{\nabla \varepsilon}{\varepsilon}\right) E \frac{\varepsilon E}{1+(\varpi \tau)^{2}}+\frac{1}{2} \varepsilon|E|^{2} \frac{\nabla \varepsilon}{\varepsilon}\right],
$$

where $E$ and $\omega$ are the magnitude and the angular frequency of the electric field, respectively. The charge relaxation time of the fluid is $\tau=\varepsilon / \sigma$, with $\sigma$ being the conductivity and $\varepsilon$ the permittivity of the medium. The first and second summands in Eq. (1) represent the Coulomb and the dielectric forces, respectively. The total force depends on (i) the strength of the electric field, (ii) its frequency, (iii) the charge relaxation time of the fluid, and (iv) the permittivity and/or conductivity gradients in the medium. These gradients are a prerequisite for force generation. They can be introduced by Joule heating in the medium-e.g., by the electric field itself [24] or by external heating elements [28]. The conductivity and permittivity gradients can be related to the temperature gradient by the partial derivatives of the two parameters over the temperature: $\nabla \sigma=\frac{\partial \sigma}{\partial T} \nabla T$ and $\nabla \varepsilon=\frac{\partial \varepsilon}{\partial T} \nabla T$. The temperature dependence of the conductivity and the permittivity of an aqueous solution at room temperature can be described by the linear temperature coefficients

$$
\frac{\partial \sigma}{\sigma \partial T}=0.02 K^{-1}, \quad \frac{\partial \varepsilon}{\varepsilon \partial T}=-0.004 K^{-1},
$$

respectively [30]. The opposite signs of the derivatives lead to opposite orientations of the field-induced Coulomb and dielectric forces in the low- and high-frequency ranges, respectively. Introducing the linear temperature coefficients into Eq. (1) leads to

$$
\left\langle F_{E}\right\rangle=-0.5 \varepsilon E^{2} \nabla T\left(\frac{0.024 K^{-1}}{1+(\varpi \tau)^{2}}-0.002 K^{-1}\right) .
$$

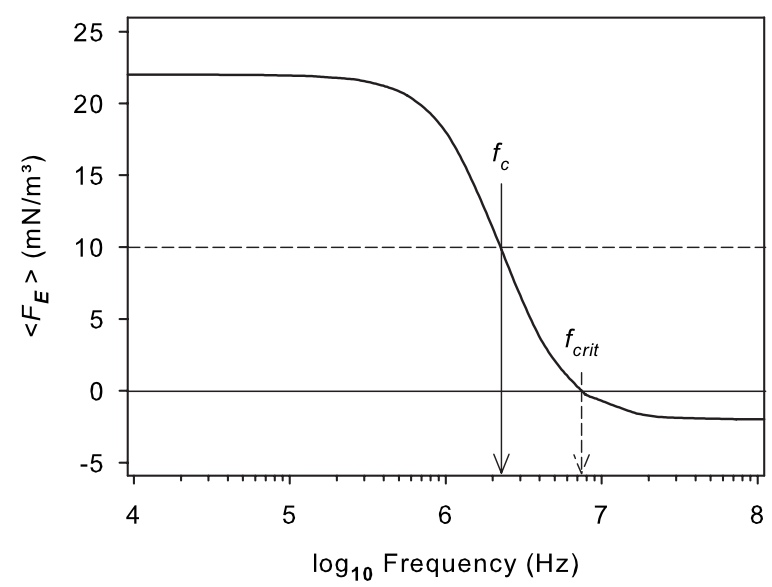

FIG. 1. Time-averaged force on a volume element as a function of frequency based on Eq. (3) for $\varepsilon_{\text {rel }}=80.2, \sigma=10 \mathrm{mS} \mathrm{m}^{-1}$, and $-0.5 \varepsilon E^{2} \nabla T=1 \mathrm{kN} \mathrm{m}^{-3}$. The charge relaxation time is $\tau=\varepsilon / \sigma=7.1$ $\times 10^{-8} \mathrm{~s}$. The arrows mark the characteristic frequency $f_{c}$ for the dispersion of the Coulomb effect and the critical frequency $f_{\text {crit }}$ at which the pump direction is reversed.

Figure 1 shows the force-frequency spectrum given by Eq. (3).

A positive force plateau is generated at frequencies below $f_{\text {crit }}$ by the dominating Coulomb forces, whereas a negative plateau is generated by the dielectric gradients at frequencies higher than $f_{\text {crit }}$. The ratio of the two force plateaus is 11:1 [Eq. (3)]. The characteristic frequency for the transition between the plateaus is determined by the time constant: $f_{c}$ $=\frac{1}{2 \pi \tau}$. The Coulomb force and the dielectric force are equal at the critical frequency $f_{\text {crit }}$, resulting in a zero net force on the fluid. Equation (3) gives $f_{\text {crit }}=\frac{\sqrt{11}}{2 \pi \tau}$. Note that the pumping force is proportional to the real part of the induced volume polarization. Accordingly, $f_{c}$ is at the midpoint of the two force plateaus. According to the temperature dependences of the conductivity and permittivity, we obtain a relaxation time

$$
\tau=\frac{\varepsilon(T)}{\sigma(T)}=\tau_{\left(T_{0}\right)} \frac{1-0.004 \Delta T_{\tau}}{1+0.02 \Delta T_{\tau}},
$$

which depends on the mean temperature increase in the pump volume, $\Delta T_{\tau}$, where $\tau_{\left(T_{0}\right)}$ is the relaxation time at room temperature $\left(T_{0}=293 \mathrm{~K}\right)$. Note that the following simplifications call for a distinction to be made between the mean temperature increase (index $\tau$ ) influencing $\tau$ and the effective temperature gradient (index $E$ ) generating the pumping force by interaction with the driving field. One possible explanation is that a symmetrical temperature peak inside the pump volume would decrease $\tau$ yet not generate any net force. In the following, Eq. (4) is used for the temperature-dependent relaxation time $\tau$.

\section{B. Electrohydrodynamic pump effect based on structural polarization}

For deriving an analytical expression, we define a pump volume $A \zeta$ assuming effective values for the cross-sectional area of the pump channel $(A)$ and the active length of the pump channel $(\zeta)$. Note that $A$ and $\zeta$ may well correspond to 
the geometry of the heated pump with $\zeta$ representing the field-electrode distance-i.e., the distance in the pump channel where the force is generated (see below). For simplicity, we assume a homogeneous electric field and a temperature gradient oriented in the direction of the channel. Accordingly, we simplify $\nabla T$ to $\frac{d T_{E}}{d \zeta}$. As a first approximation, $\frac{d T_{E}}{d \zeta}$ can be obtained from $\frac{\Delta T_{E}}{\zeta}$ - i.e., the effective temperature difference per active channel length, $\zeta$. The effective temperature difference allows for an asymmetric volume polarization of the pumping medium and the generation of a pumping force from the interaction with the driving field.

Neglecting nonlinear temperature effects in the coupling of field and induced forces, the mean force $\left\langle\overline{F_{E}}\right\rangle$ acting on all volume elements in the pump volume $A \zeta$ is

$$
\left\langle\overline{F_{E}}\right\rangle=-0.5 \varepsilon E^{2} \frac{\Delta T_{E}}{\zeta}\left(\frac{0.024 K^{-1}}{1+(\varpi \tau)^{2}}-0.002 K^{-1}\right) .
$$

Multiplication with the pump volume $A \zeta$ yields the overall pump force $F_{P}=A \zeta\left\langle\overline{F_{E}}\right\rangle$ and the pump pressure as

$$
\Delta P_{p}=\frac{F_{P}}{A}=\zeta\left\langle\overline{F_{E}}\right\rangle=-0.5 \varepsilon E^{2} \Delta T_{E}\left(\frac{0.024 K^{-1}}{1+(\varpi \tau)^{2}}-0.002 K^{-1}\right) .
$$

For frequencies below $f_{c}$, Eq. (6) can be simplified to

$$
\Delta P_{p}=-\left(0.011 K^{-1}\right) \varepsilon E^{2} \Delta T_{e} .
$$

The pump plateau at very high frequencies is

$$
\Delta P_{p}=\left(0.001 K^{-1}\right) \varepsilon E^{2} \Delta T_{e} .
$$

In the analytical model, the pump pressure $\Delta P_{p}$ and the flow velocity $v$ depend linearly on $\Delta T$ and quadratically on $E$. Interestingly, the geometric parameters are canceled out, which suggests that our pumping principle is freely scalable.

In operation, the pumping force must be at equilibrium with the viscous drag forces. The maximum flow velocity can be correlated to the pump pressure by a linear relation

$$
v=\frac{\phi}{\eta} \Delta P_{P},
$$

where $\eta$ is the viscosity of the fluid, while $\phi$ is the specific geometry coefficient of a certain channel geometry ( $\phi$ is a complex function of $\zeta$ and $A$; see Appendix A). Finally, we obtain the maximum flow velocity

$$
v=-\frac{0.5 \phi \varepsilon E^{2} \Delta T_{E}}{\eta}\left(\frac{0.024 K^{-1}}{1+(\varpi \tau)^{2}}-0.002 K^{-1}\right) .
$$

\section{MATERIAL AND METHODS}

\section{A. Structure of the field gradient pump}

The field gradient pump was a sandwich structure comprised of two glass layers (microscopic cover slips) on a microscopic slide (Fig. 2). The pump structure was carved by hand into the center layer using grinding and milling tools. The structure consisted of two reservoirs (length, $11.6 \mathrm{~mm}$; width, $1.7 \mathrm{~mm}$; height, $0.52 \mathrm{~mm}$ ) on both sides of a partitioning wall. The reservoirs were connected by two funnel-

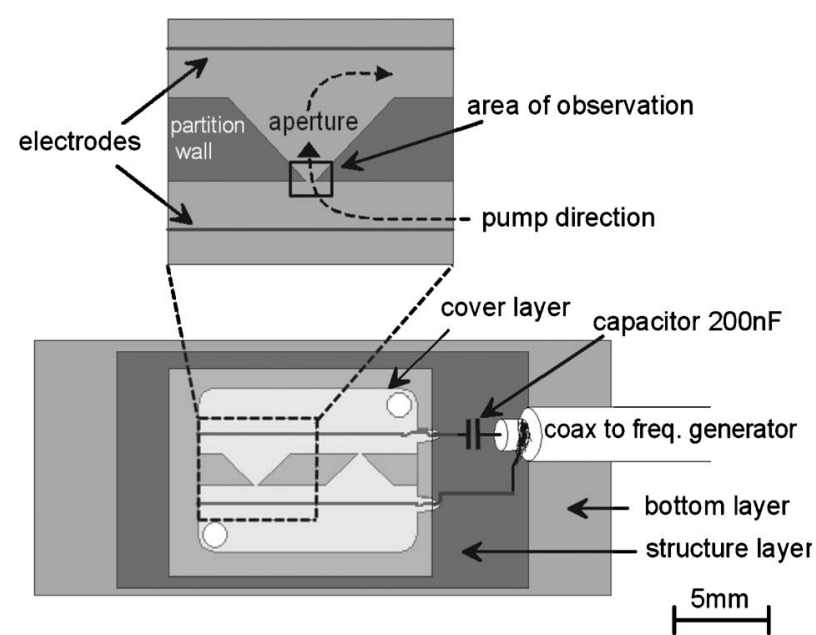

FIG. 2. Scheme of the field gradient pump structure.

shaped apertures pointing in opposite directions, thus allowing for circular fluid flow in the pump. Each aperture measured $3.6 \mathrm{~mm}$ at the entrance and $0.2 \mathrm{~mm}$ at the funnel tip. Field electrodes consisted of two platinum wires spanning the reservoirs in parallel to the partitioning wall with $1 \mathrm{~mm}$ separation from the wall. The pump structure was sealed using epoxy glue. Finally, two filling nozzles were mounted above two bore holes in the top layer for pump filling and fluid exchange.

\section{B. Structure of the heated pump}

The heated pump structure was produced photolithographically on a thin glass wafer (thickness $550 \mu \mathrm{m}$ ) by GeSim GmbH, Grosserkmannsdorf, Germany. The wafer glass carried the platinum structures (thickness approximately $110 \mathrm{~nm}$ ) of the field electrodes and heating elements (Fig. 3).

A photoresist layer of approximately $50 \mu \mathrm{m}$ in thickness formed the fluid channel wall structures. For measurements, the channels were closed by a polydimethylsiloxane (PDMS) cover carrying the filling nozzles. The pump chips fitted the GeSim's MicCell-monitoring system.

The heated pump had two reservoirs (length, $1100 \mu \mathrm{m}$; width, $350 \mu \mathrm{m}$; height, $50 \mu \mathrm{m})$ connected by two channels of $1000 \mu \mathrm{m}$ length, $100 \mu \mathrm{m}$ width, and $50 \mu \mathrm{m}$ height (Fig.



FIG. 3. Scheme of the heated pump structure. Arrows show the pump direction. Heating connectors and field electrodes $(E 1, E 2)$ are designated. 
3). One channel contained the electrodes (electrode channel), while the other was used to monitor the fluid velocities (observation channel). This design allowed for a circular flow in the pump structure. The electrode channel contained two parallel-field electrodes (electrode width, $100 \mu \mathrm{m}$; distance, $390 \mu \mathrm{m})$ and a heating element located next to electrode $E 2$. The heating element was a $30-\mu \mathrm{m}$-broad meander-shaped filament spanning the electrode channel 6 times $[28,29]$.

\section{Operation of the micropumps}

A HP 8116A generator (Agilent Technologies, Santa Clara, CA, USA) drove the field electrodes of all pumps via a coaxial cable. A capacitor $(200 \mathrm{nF})$ was switched in series to the signal wire to block dc. The generator provided a sinusoidal signal in the range from $1 \mathrm{~Hz}$ to $52 \mathrm{MHz}$ at up to $12 \mathrm{~V}_{\mathrm{rms}}$. An amplifier (ENI 350L, ENI, Rochester, NY, USA) was used occasionally to increase the generator signal up to $80 \mathrm{~V}_{\mathrm{rms}}$ in the frequency range from $3 \mathrm{kHz}$ to $34 \mathrm{MHz}$. The heating filament of the heated pump was connected to a dc source. The field electrode $E 2$ and the adjacent heating filament contact were connected to ground. A resistor of $1 \mathrm{k} \Omega$ was switched in series to the heating filament in order to limit the dc heating current. The power uptake of the heating system was calculated from the measured heating current and the dc source voltage.

For measurements, the field gradient pump was filled carefully by hand using syringes and silicon tubing, while the heated pump was filled automatically by a microdispenser (GeSim GmbH, Grosserkmannsdorf, Germany). After filling, the pumps were pinched off above the filling nozzles to block hydrostatic fluid motions.

$\mathrm{NaCl}$ solutions of different conductivities were used. Fluid conductivities ranged from 1 to $112 \mathrm{mS} \mathrm{m}^{-1}$. Latex particles (Serva Electrophoresis GmbH, Heidelberg, Germany, diameter $940 \mathrm{~nm}$ ) were added as optical tracers for microscopic velocity detection. Before each measurement the pumps were rinsed several times with the new fluid. Latex-tracer velocities were detected in the funnel tip (Fig. 2) and the observation channel (Fig. 3) in the field gradient pump and in the heated pump, respectively. The velocities of single latex particles were determined on a video monitor using an ocular grid and a stopwatch. Grid distances of $25 \mu \mathrm{m}$ and $125 \mu \mathrm{m}$ were used to measure different tracer velocities. The velocity was measured for each condition for at least three different particles.

\section{Numerical modeling of the micropumps}

Two-dimensional (2D) models were developed for the pumps using the finite-element program COMSOL MULTIPHYSICS [34]. For this, we employed the modules for electrostatic, general heat transfer and laminar fluid flow (see Appendix B). Appropriate equations for the temperature dependences of the medium properties were introduced into the modules and applied to Eq. (1). The specific physical properties of water and glass were assigned to the pump medium and the solid structure. The platinum structures were modeled as equipotential areas with aqueous properties to allow for fluid flow across electrodes and heating elements in the 2D model.

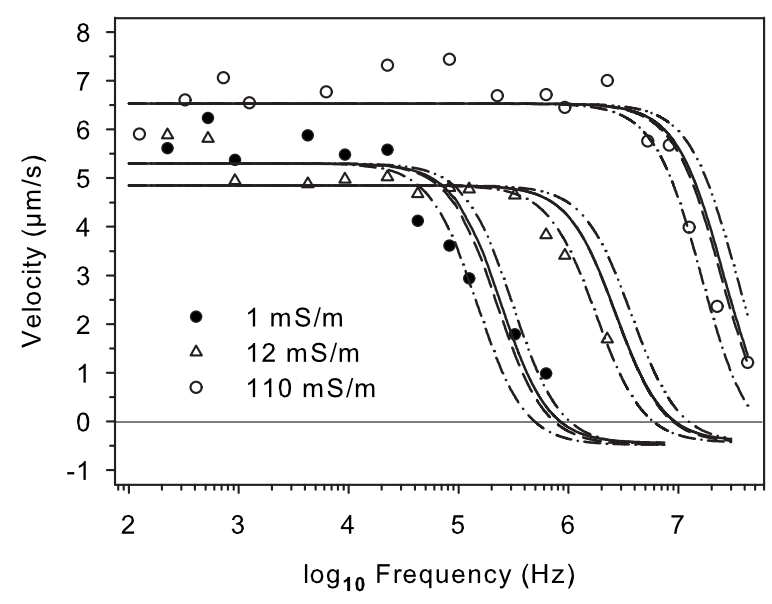

FIG. 4. Field gradient pump: velocity spectra at electrode voltages of $12 \mathrm{~V}_{\text {rms }}$ assuming a $\varepsilon_{\text {rel }}$ of 80.2. The analytical equation (11) was fitted to data of three different conductivities assuming a $\Delta T_{\tau}$ of $0 \mathrm{~K}$ (dashed curves). Dash-dotted and dash-double-dotted curves assume $\Delta T_{\tau}$ of $-15 \mathrm{~K}$ and $+15 \mathrm{~K}$. Curves of the numerical model (solid lines) were normalized to the fitted low-frequency plateaus. Note the slight shifts caused by a temperature increase of approximately $2 \mathrm{~K}$ inherent to the model (see Discussion).

\section{RESULTS}

\section{A. Field gradient pump}

After applying $12 \mathrm{~V}_{\text {rms }}$ to the electrodes, the fluid was pumped in a circular pattern, directed against the funnelshaped apertures of the partitioning wall. The flow instantaneously started when the electrode potential was switched on and stopped after switched off. Pumping velocities exhibited a plateau in the low-frequency range and a characteristic dispersion in accordance with Eq. (3) (Fig. 4). The characteristic dispersion frequency $f_{c}$ increased with increasing conductivity (compare to Figs. 1 and 4). However, we could not observe a reversal of the pump flow at high frequencies as predicted by the theory.

The field strength dependence was measured with increasing electrode potentials at $300 \mathrm{kHz}$ and a fluid conductivity of $34 \mathrm{mS} \mathrm{m}^{-1}$ (Fig. 5). We found an almost linear pumpvelocity increase for electrode potentials from 5 to $28 \mathrm{~V}_{\text {rms }}$. Nevertheless, the best fit was a power function of the driving voltage with an exponent of approximately 1.3 [velocity $\left.=1.1(\text { voltage })^{1.3}\right]$.

\section{B. Heated pump}

No pumping was observed when the heating or the ac pump voltages were applied separately. An instantaneous start of the pump flow could be observed when both voltages were applied, either after a consecutive or synchronous switch-on. The pump flow was directed from the heating filament to the field electrodes for frequencies below $f_{c}$ and reversed at higher frequencies. Velocity spectra were measured at fluid conductivities of $14,24,70$, and $112 \mathrm{mS} \mathrm{m}^{-1}$ (Fig. 6) with an electrode potential of $40 \mathrm{~V}_{\mathrm{rms}}$. The power uptake of the heating system was adjusted to $0.18 \mathrm{~W}$. Maximum fluid velocities of $60-130 \mu \mathrm{m} \mathrm{s}^{-1}$ were recorded for frequencies below $f_{c}$. The velocity spectra were similar to 


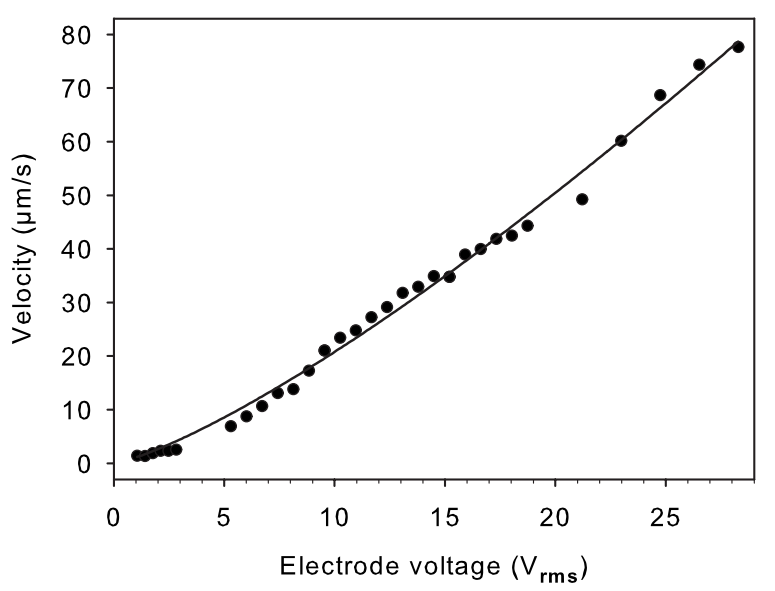

FIG. 5. Field gradient pump: velocity as a function of applied electrode potential for $\sigma=34 \mathrm{mS} \mathrm{m}^{-1}$ and a field frequency of $300 \mathrm{kHz}$. Solid line: fitted function [velocity $=1.1(\text { voltage })^{1.3}$.

those of the field gradient pump as $f_{c}$ increased with increasing conductivities.

In contrast to the field gradient pump, we observed a reversed pumping direction at frequencies above $f_{\text {crit }}$ (Fig. 6, conductivities of 14 and $24 \mathrm{mS} / \mathrm{m}$ ). The low reversepumping velocities correspond to the theory (Fig. 1). No reverse flow was measured for the conductivities of 70 and $112 \mathrm{mS} \mathrm{m}^{-1}$. At these conductivities, a reversed pump direction was expected above the theoretical $f_{\text {crit }}$ of 68 and $106 \mathrm{MHz}$. Nevertheless, these frequencies were above the frequency range of the generator.

The heating power and electrode potential dependencies of the pump velocity were measured at fixed frequencies at three different conductivities (Fig. 7). The electrode potential was fixed at $40 \mathrm{~V}_{\text {rms }}$ to measure the heating power dependence. A linear velocity increase was obtained with similar slopes at all conductivities. The velocity dependence on the electrode potential was determined in the voltage range from 15 to $60 \mathrm{~V}_{\text {rms }}$ for a fixed heating power uptake of $0.18 \mathrm{~W}$ (Fig. 8). The best fit was a power function with an exponent between 1.8 and 1.9 .

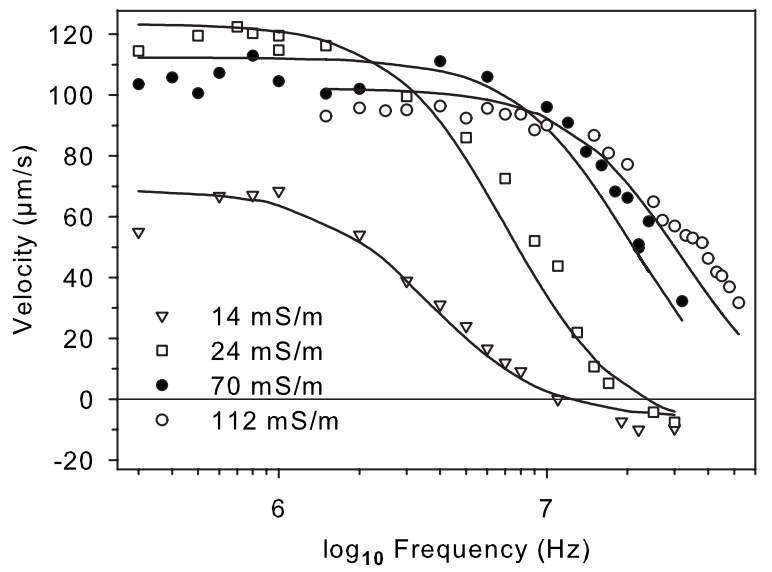

FIG. 6. Heated pump: velocity as a function of frequency for different conductivities. Fit with analytical model [Eq. (10); see Discussion]. Electrode potentials were $40 \mathrm{~V}_{\mathrm{rms}}$. The power uptake of the heating system was $0.18 \mathrm{~W}$.

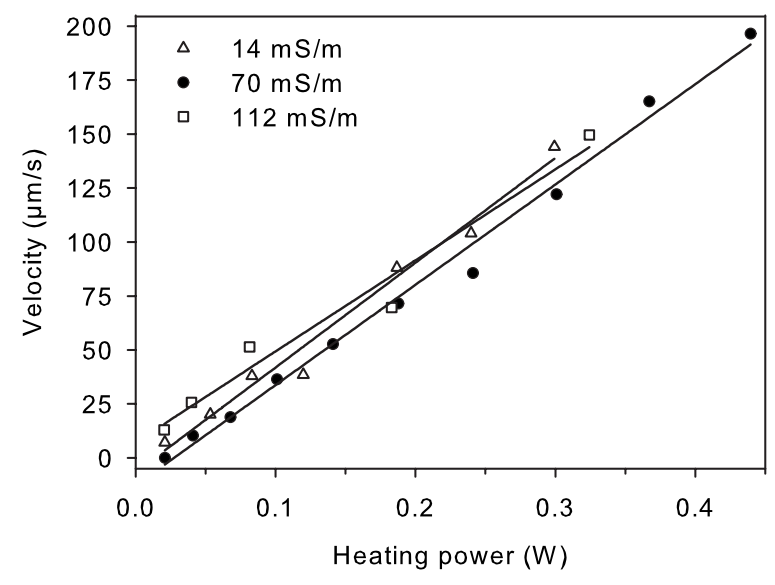

FIG. 7. Heated pump: velocity as a function of power uptake of the heating system. Electrode potentials were $40 \mathrm{~V}_{\mathrm{rms}}$; frequencies were $300 \mathrm{kHz}$ for conductivities of 14 and $70 \mathrm{mS} \mathrm{m}^{-1}$ and $1 \mathrm{MHz}$ for $112 \mathrm{mS} \mathrm{m}^{-1}$.

\section{DISCUSSION}

\section{A. Field gradient pump}

The numerical model of the field gradient pump has been solved for the three experimental conductivities $(\sigma=1,12$, and $110 \mathrm{mS} / \mathrm{m})$ in the frequency range from $200 \mathrm{~Hz}$ to $100 \mathrm{MHz}$. In all cases, temperature and field maxima are located in the narrowest regions of the funnel. Nevertheless, the funnel shape causes asymmetric field and temperature distributions. As a result, the volume forces at both sides of the funnel tip do not necessarily cancel each other out. This imbalance leads to a net flow in one direction [Fig. 9(B)].

In the following, we compare experimental and numerical velocity spectra with our analytical model [Eq. (10), Fig. 4]. The frequency dependence of the pump force is governed by the terms in parentheses of Eq. (3). The frequencyindependent parameters-field strength, temperature difference, viscosity, and specific geometry coefficient in Eq. (10) — can be summarized by the coefficient $\gamma$ :

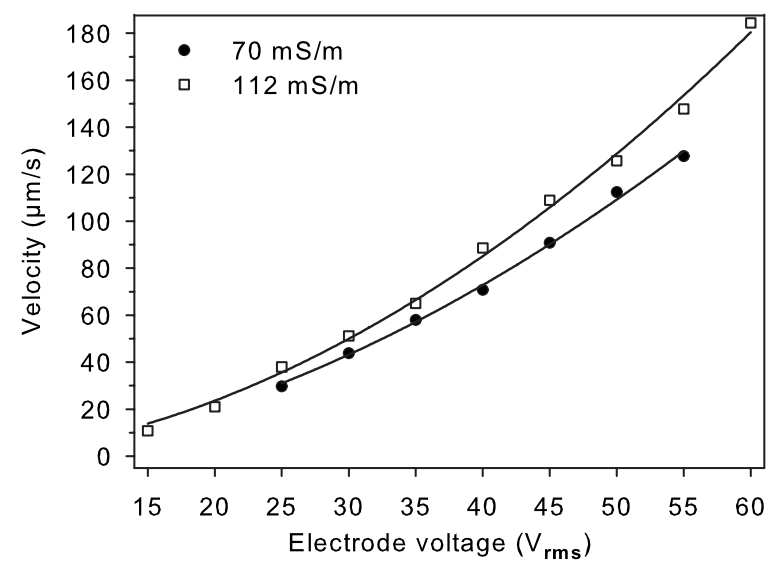

FIG. 8. Heated pump: velocity as a function of applied electrode potential. Frequencies were $300 \mathrm{kHz}$ and $1 \mathrm{MHz}$ for conductivities of 70 and $112 \mathrm{mS} \mathrm{m}^{-1}$, respectively. The power uptake of the heating system was $0.18 \mathrm{~W}$. 

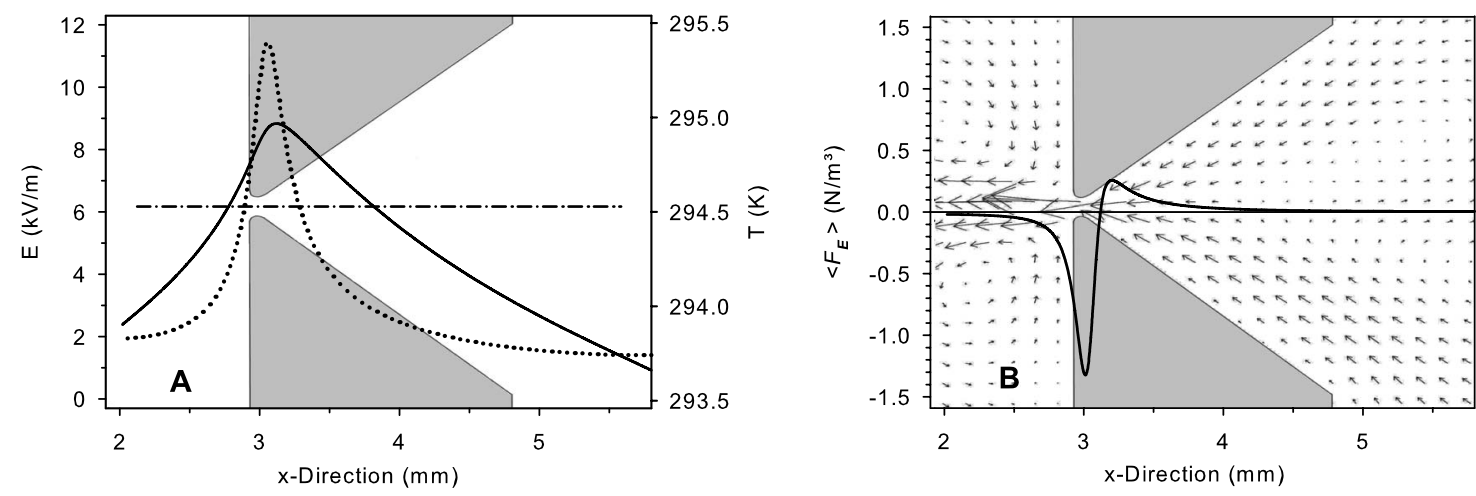

FIG. 9. Geometry of the field gradient pump. (A) Plots of the electric (dotted line) and temperature fields (solid line) along the symmetry line (dash-dotted line) for $110 \mathrm{mS} / \mathrm{m}$ and $100 \mathrm{kHz}$. (B) The resulting volume force in the $x$ direction (solid line). The arrows show the resulting velocity field. $\varepsilon_{r e l}$ at $T_{0}$ and the electrode voltage were assumed to be 80.2 and $12 \mathrm{~V}_{\text {rms }}$ (for more details, see Appendix B).

$$
v=-\gamma\left(\frac{0.024 K^{-1}}{1+(\varpi \tau)^{2}}-\frac{0.004 K^{-1}}{2}\right)
$$

This simplified equation has been used to fit the experimental velocity data of Fig. 4 with $\gamma$ as the only free variable. $\tau_{\left(T_{0}\right)}$ was given by equation (4) for $\Delta T_{\tau}=0 \mathrm{~K}$.

After fitting the analytical equation (11), the velocity spectra of the numerical model were normalized to the obtained low-frequency plateau heights (Fig. 4). This was necessary because the numerical 2D model underestimates the friction (top and bottom friction are neglected) and therefore overestimates the pump velocity, even though the frequency dependence of the force is largely correct (see heated pump). As can be seen from Fig. 4, the analytical and numerical models correspond quite well after normalization. The heating inherent in the numerical model causes a conductivity increase and a slightly shorter $\tau$ than in the analytical model with fixed temperature. In both cases, the slight deviation from the experimental spectra seems to stem from too short a relaxation time $\tau$. Another contribution to the deviations might be a broadening of the dispersion by a $\tau$ distribution resulting from the temperature and field distributions in the pump volume. Interestingly, a higher relaxation time $\tau$ would require a temperature decrease which cannot easily be explained (compare to the $-15 \mathrm{~K}$ and $+15 \mathrm{~K}$ curves in Fig. 4).

In the field gradient pump, a number of experimental observations are inconsistent with the theory. Possible reasons could be the following: (i) we did not observe a reversal of the pump direction at high frequencies; (ii) the pump direction observed was opposite to the pump direction of the numerical model [compare pump directions in Figs. 2 and 9(B)]. The numerical model (data not shown) and Eq. (3) predict a pump velocity proportional to $E^{4}$ and $\sigma$. In contrast, the measurements show (iii) a dependence on $E^{1.3}$ and (iv) no significant change in the plateau velocities for different conductivities.

Concerning (i), the absence of a reverse pumping at high frequencies might be due to the detection limit of the velocity measurements and/or electronic problems. For the geometrical pump, the theoretical reverse velocity of $0.6 \mu \mathrm{m} \mathrm{s}^{-1}$ is hard to distinguish from the Brownian motion of the latex tracers. The capacitive and resistive load of the chamber and the inductances of the connecting wires form a low pass, causing a field strength decrease at higher frequencies [32]. Nevertheless, any influence of the lattices' dielectrophoretic displacement on the fluid velocity may be neglected, because the modeled electric field gradient at the funnel tip would only allow for a maximum dielectrophoretic velocity of $0.06 \mu \mathrm{m} \mathrm{s}^{-1}$ for $1-\mu \mathrm{m}$ particles.

Concerning (ii), we assume that minor disturbances at the funnel tip might cause an alteration of the flow direction because of the fragile imbalance of the volume forces at both sides of the funnel tip [compare the pump direction in Fig. 2 to Figs. 9(A) and 9(B)]. The process used to manufacture the pump gave the chamber walls a coarse surface, which may cause high and irregular local electric field gradients, especially at the funnel tip. Such local gradients may generate local convections that initiate pumping in a certain direction. Electrothermal fluid flow induced by the microscope light, as described by Green et al. [31], is another possible disturbance. We found a similar influence of microscopic light on other micropump structures. Domination of external heating at either side of the funnel tip may cause the reversal of the pump direction. In the case that external heating dominates the temperature field, the velocity will be proportional to $E^{2}$.

Concerning (iii), the predicted pump velocity dependence on $E^{4}$ is contrary to the measured dependence on $E^{1.3}$. So far, we cannot explain this observation. Reasons that may contribute are the counteracting volume forces at both sides of the funnel tip (which should both depend on $E^{4}$ ) and a possible role of external heating (see above). It is likely that not only the temperature gradient, but also the field gradient need to be taken into account in order to achieve a full analytical description.

Concerning (iv), for a nonmoving fluid, the Jouletemperature increase should depend linearly on the conductivity of the medium. Nevertheless, the linear dependence will be reduced by a faster medium exchange; i.e., an increase in pump velocity will diminish the effective temperature gradient and thus, in turn, the pump velocity. As a result, the conductivity dependence of the velocity will be reduced (cf. Table I).

\section{B. Heated pump}

The heated pump was modeled numerically for two different states, with and without external heating by the heat- 
TABLE I. Heated pump: fitted $\Delta T, f_{c}$, and $f_{c r i t}$ for different fluid conductivities $\sigma$.

\begin{tabular}{cccccc}
\hline \hline $\begin{array}{c}\sigma \\
\left(\mathrm{S} \mathrm{m}^{-1}\right)\end{array}$ & $\begin{array}{c}\Delta T \\
(\mathrm{~K})\end{array}$ & $\begin{array}{c}f_{c} \\
(\mathrm{MHz})\end{array}$ & $\begin{array}{c}f_{\text {crit }} \\
(\mathrm{MHz})\end{array}$ & $\begin{array}{c}\Delta P_{P} \\
\left(\mathrm{~N} \mathrm{~m}^{-2}\right)\end{array}$ & $\begin{array}{c}Q \\
\left(\mathrm{nl} \mathrm{min}^{-1}\right)\end{array}$ \\
\hline 0.014 & 7.3 & 3.7 & 12.1 & 0.60 & 10.4 \\
0.024 & 13.0 & 7.1 & 23.4 & 1.07 & 18.6 \\
0.070 & 11.9 & 20.6 & 68.2 & 0.98 & 16.9 \\
0.112 & 10.8 & 31.8 & 105.5 & 0.89 & 15.4 \\
\hline \hline
\end{tabular}

ing filament (Fig. 10). When the heating filament was turned off and the electrode potential was set to $40 \mathrm{~V}_{\text {rms }}$, a symmetric temperature field was generated between the electrodes [Fig. 10(A)]. Even though field absorption leads to a temperature gradient near the electrodes, the symmetric volume forces cancel out along the channel [Fig. 10(B)]. The superposition of field-induced heat dissipation (Joule's heat) and direct heating by the heating filament lead to an asymmetric temperature distribution between the electrodes when the heating filament was switched on. The result was a pump force with a direction according to our theoretical prediction [arrows in Fig. 10(B)].

The simple design of the heated pump allowed us to develop an analytical model introducing the specific geometry coefficient for rectangular channel geometry [Eq. (A4)] and the temperature difference between the electrodes $\Delta T_{E}$ into Eq. (10):

$$
v_{e o}=-\frac{2.85 \times 10^{-10}}{\eta 2.47 L_{e o}} 0.5 \varepsilon E^{2} \Delta T_{E}\left(\frac{0.024 K^{-1}}{1+(\varpi \tau)^{2}}-0.002 K^{-1}\right),
$$

with $\eta$ being the fluid viscosity and $L_{e o}$ being the length of the electrode and observation channels.

Equation (12) was fitted to the velocity spectra (Fig. 6) using $\Delta T$ as the only free variable. For the sake of simplicity, we assumed $\Delta T=\Delta T_{E}=\Delta T_{\tau}$ [see Eq. (4)]. A field strength of $E=102.56 \mathrm{kV} \mathrm{m}^{-1}(40 \mathrm{~V}$ per $390 \mu \mathrm{m}$ electrode distance $)$ and a constant viscosity of $\eta=1 \times 10^{-3} \mathrm{~N} \mathrm{~s} \mathrm{~m}^{-2}$ were assumed (note that most of the fluid volume in the circular channel is at room temperature). The fitted values of $\Delta T$ and the corresponding $f_{c}$ and $f_{c r i t}$ are presented in Table I.

Experimental velocity spectra are largely consistent with the model (Fig. 6). Both $f_{c r i t}$ and $f_{c}$ increase linearly with the medium conductivity. Experimentally, the external heating power was kept constant. External heating dominated the temperature field, even though Joule heating increased at higher medium conductivities. $\Delta T$ values between 7.3 and $13.0 \mathrm{~K}$ were obtained, corresponding to $\Delta P_{p}$ plateaus ranging from 0.6 to $1.1 \mathrm{~N} \mathrm{~m}^{-2}$ and volume flow rates $Q$ between 10 and $19 \mathrm{nl} \mathrm{min}^{-1}$. For the whole electrode-channel volume of $5 \mathrm{nl}$, the self-pumping frequency was $2-4 \mathrm{~min}^{-1}$.

The linear and quadratic dependences on $\Delta T$ and $E$, respectively, are consistent with the pump velocity measurements as a function of heating power and electrode potential [Eq. (6), Figs. 7 and 8]. Nevertheless, the power functions fitted to the experimental data show a coefficient slightly lower than 2. For a constant power input from the heating element, the increasing heat dissipation in between the field electrodes may lead to a reduction of the increase in the asymmetry of the temperature distribution $\left(\Delta T_{E}\right)$ with increasing medium conductivity. Another possible reason is discussed in chapter Sec. V A, point (iv). In general, $E$ and $\Delta T_{E}$ are limited by the critical temperature maximum in the electrode channel. At high medium conductivities, a temperature peak may be observed in between the field electrodes. In this case, the induced forces at both sides of the peak exhibit opposite directions. In addition, $\Delta T_{E}$ and $\Delta T_{\tau}$ will be separated, leading to an increasing error in the fitted $\Delta T$ values.

In the following, we compare the pumping forces obtained from the analytical and the numerical models. The dimensional restrictions of the numerical 2D model lead to an underestimation of friction and an overestimation of the fluid velocity, which can therefore not directly be compared to experimental values (as in the case of the field gradient pump). Nevertheless, the electric field and the temperature field are modeled sufficiently well to allow for the calcula-
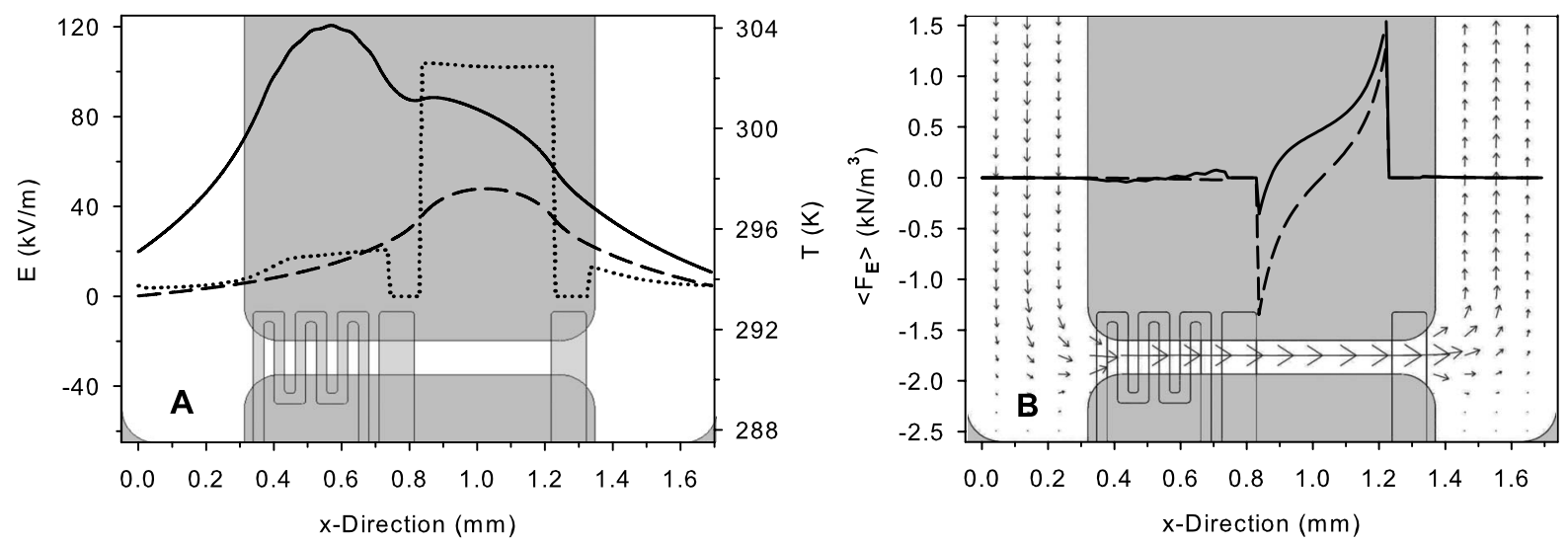

FIG. 10. The geometry of the heated pump. (A) Plots of the electric field (dotted line) and the temperature field with (solid line) and without (dashed line) heating. (B) The volume force in the $x$ direction with (solid line) and without (dashed line) heating along the central cross section of the pump channel. The arrows show the velocity field. Input parameters: $\varepsilon_{\text {rel }}=80.2 ; \sigma=0.024 \mathrm{~S} \mathrm{~m}^{-1}$; electrode potential, $40 \mathrm{~V}_{\mathrm{rms}}$; field frequency, $1 \mathrm{MHz}$. For further details, see Appendix B. 


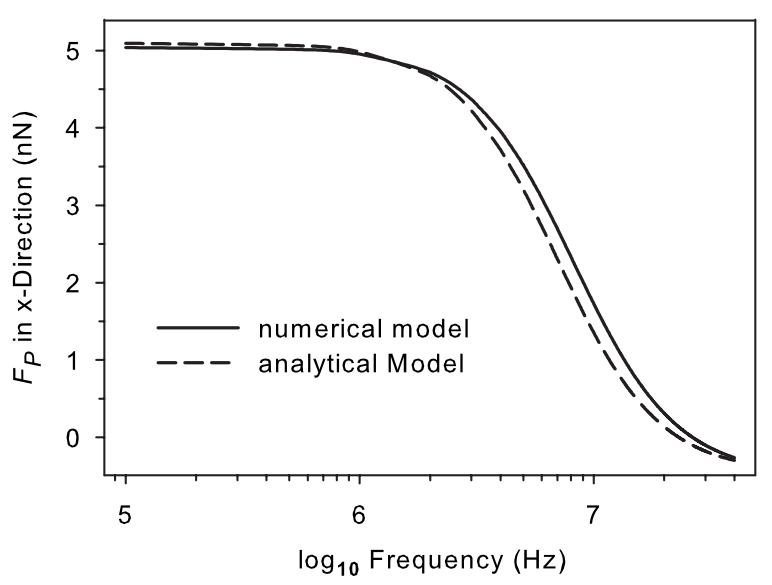

FIG. 11. Spectra of the pumping force $F_{P}$, calculated from the analytical and the numerical models. Input parameters of $\varepsilon_{\text {rel }}$ $=80.2, \sigma=0.024 \mathrm{~S} \mathrm{~m}^{-1}, E=102.5 \mathrm{kV} \mathrm{m}^{-1}$, and $\Delta T$ of $13.4 \mathrm{~K}$ between the electrodes were used.

tion of a mean volume force $\left\langle\overline{F_{E}}\right\rangle$ from the 2D model (see Fig. 10). This force can be normalized to the height of the electrode channel of $50 \mu \mathrm{m}$. The resulting pumping force $F_{P}$ was compared with Eq. (6). As can be seen in Figs. 6 and 11, the experimental force spectra and the numerical and analytical models are largely consistent, proving the applicability of the analytical model. The low-frequency plateaus deviate by only $1.1 \%$ (Fig. 11 ).

In [28] we tested a different design of the heated pump. In this design, the counterfield electrode was missing and the heating element was used as the second ac field electrode. The improved superposition of the two heat sources increased $\Delta T_{e}$ and thus increased the pump efficiency. Nevertheless, an open question was that we did not find the expected velocity plateau in the low-frequency range. Instead, the pump velocities increased with frequency, exhibiting a peak around $20 \mathrm{MHz}$. The results presented here and new impedance measurements (unpublished data) have proven that this increase was caused by a frequency-dependent bridging of the passivation layer of the platinum electrodes rather than a frequency dependence in the electrohydrodynamic pump effect. We could also show that heated pumps can be applied at fluid conductivities above $1.3 \mathrm{~S} \mathrm{~m}^{-1}$. Accordingly, they can be used to pump cell culture solutions in microfluidic or cell chip systems.

\section{CONCLUSIONS}

The advantages of the presented dynamic field pumps are (i) their simple design, (ii) a force generation throughout the volume of the pump medium, (iii) the operation by ac fields avoiding electrochemical electrode deterioration and/or the release of toxic products, (iv) the usability in a wide conductivity range of aqueous or other solutions, and (v) the constant pump velocity in a wide frequency range allowing for frequency adjustments for specific purposes such as the combination of fluid pumping with electrokinetic particle manipulation. Of the two pump designs, we would favor the heated pump for its higher efficiency. There, the volume

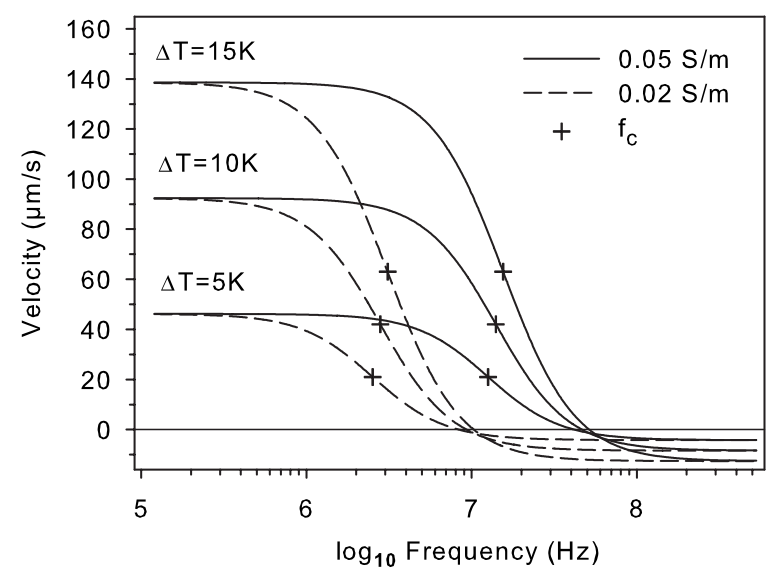

FIG. 12. Modeled velocity as a function of frequency for two medium conductivities and the following parameters: $\varepsilon_{\text {rel }}=80.2, E$ $=102.5 \mathrm{kV} \mathrm{m}^{-1}, \eta=10^{-3} \mathrm{~N} \mathrm{~s} \mathrm{~m}^{-2}, L_{\mathrm{eo}}=1.0 \times 10^{-3} \mathrm{~m}, \sigma=0.05$ or $0.02 \mathrm{~S} \mathrm{~m}^{-1}$, and $\Delta T=5,10$, or $15 \mathrm{~K}$.

forces point predominantly in one direction only. The operation is more reliable and its properties are consistent with the numerical model as well as the analytical description. In [28] we tested a different design of the heated pump. In this design, the counterfield electrode was missing, using the heating element as the second ac field electrode. The improved superposition of the two heat sources increased $\Delta T_{e}$ and thus increased the pump efficiency. In addition, we applied heated pumps at fluid conductivities above $1.3 \mathrm{~S} \mathrm{~m}^{-1}$ [28]. Accordingly, they are appropriate to pump cell culture solutions in microfluidic ( $\mu$ TAS) or cell chip (Lab-on chip) systems. Nevertheless, the advantage of the field gradient pump is its extremely simple design. A clear disadvantage is the narrowing, funnel-shaped pump channel. Volume forces of similar magnitude are created on both sides of the funnel, reducing the pumping efficiency and resulting in a not fully predictable pumping behavior. Improved channel geometry might create an unambiguous pump direction and in turn increase the pumping efficiency. Future developments will reveal which of the two principles of inducing temperature gradients in the pumping medium is superior: either inherent field (Joule) heating or separate heating by external elements. In any case, the advantage of both types is the induction of a pumping force throughout the volume and not only in thin layers of the pumping medium, like it is induced in electroosmotic or traveling-wave pumps. Certainly, the efficiency of the presented dynamic field pumps can be increased by improved designs.

\section{ACKNOWLEDGMENTS}

We are grateful to Jutta Donath and Derk Wachner for assistance and technical support. We wish to thank Prof. Dr. Arzhang Khalili for helpful comments and Robert Sleigh for help with the manuscript.

\section{APPENDIX A}

The pressure drop $\Delta P$ over the channel length $L$ is related to the volume flow rate $Q$ by 
TABLE II. Summary of variables and input values for the numerical model.

\begin{tabular}{|c|c|c|}
\hline Symbol & Variable & Remarks \\
\hline$d_{z}$ & Thickness of structure & See text \\
\hline$h_{u}, h_{d}$ & $\begin{array}{l}\text { Heat transfer coefficient, } \\
\text { upside and downside }\end{array}$ & $40 \mathrm{~W} \mathrm{~m}^{-2} \mathrm{~K}^{-1}$ \\
\hline$k$ & Thermal conductivity & $\begin{array}{l}\text { Fluids: } T \text { dependent (see [30]), solids: } \\
1 \mathrm{~W} \mathrm{~m}^{-1} \mathrm{~K}^{-1}\end{array}$ \\
\hline$n$ & Normal unit vector & \\
\hline$C_{p}$ & Heat capacity & $\begin{array}{l}\text { Fluids: } T \text { dependent (see [30]), solids: } \\
703 \mathrm{~J} \mathrm{~kg}^{-1} \mathrm{~K}^{-1}\end{array}$ \\
\hline$F$ & Volume force field & See Eq. (1) \\
\hline$I$ & $\begin{array}{l}\text { Identity matrix or unit } \\
\text { diagonal matrix }\end{array}$ & \\
\hline$J$ & Current density & \\
\hline$Q$ & Heat source & $\begin{array}{l}\text { Solved by electrostatic generalized and conductive } \\
\text { media dc modules }\end{array}$ \\
\hline$Q_{j}$ & Current source & Was set to zero \\
\hline$T$ & Temperature & Solved by general heat transfer module \\
\hline$T_{e x t, u}, T_{e x t, d}$ & $\begin{array}{l}\text { External temperature, } \\
\text { upside and downside }\end{array}$ & Set to $T_{0}=293 \mathrm{~K}$ \\
\hline$V_{r m s}, V_{d c}$ & Electric potential & $\begin{array}{l}\text { Electric potentials applied to the electrodes or the } \\
\text { heating element }\end{array}$ \\
\hline$\varepsilon_{0}$ & Permittivity of vacuum & $8.854 \times 10^{-12}$ As V m$^{-1}$ \\
\hline$\varepsilon_{r}$ & Relative permittivity & Fluids: $T$ dependent (see theory), solids: 6.7 \\
\hline$\eta$ & Dynamic viscosity & $10^{-3} \mathrm{~N} \mathrm{~s} \mathrm{~m}^{-2}$ \\
\hline$\sigma$ & Conductivity & $\begin{array}{l}\text { Fluids: } T \text { dependent (see theory), solids: } \\
\text { deactivated }\end{array}$ \\
\hline$\rho$ & Density & $\begin{array}{l}\text { Fluids: } T \text { Dependent (see [30]), solids: } \\
2203 \mathrm{~kg} \mathrm{~m}^{-3}\end{array}$ \\
\hline
\end{tabular}

$$
Q=\frac{w h^{3} \Delta P}{12 \eta L}\left(1-\frac{6\left(2^{5}\right) h}{\pi^{5} w}\right)
$$

for a laminar fluid flow through a rectangular channel of height $h$ and width $w$, where $\eta$ is the viscosity of the fluid [9]. In the heated pump, we have to consider two different geometries: the electrode-observation channels and the two reservoir channels (see Fig. 3). Given the circular pump structure, $Q$ in all channels must be the same and we can derive the relative pressure drops along the two channel geometries from Eq. (A1) by substituting the respective geometry values $\frac{\Delta P_{e o}}{\Delta P_{r e s}}=4.22$. The indices $e o$ and res stand for the electrode and observation channels as well as the reservoir channels, respectively. The total pressure drop in the pump is the sum of the particular channel pressure drops $\Delta P_{\text {tot }}$ $=2 \Delta P_{e o}+2 \Delta P_{\text {res }}$, which can be simplified to $\Delta P_{t o t}$ $=2.47 \Delta P_{e o}$. Within the rectangular observation channel of length $L_{e o}$, the pressure drop $\Delta P_{e o}$ causes a maximum flow velocity $v_{e o}$, expressed as [33]

$$
v_{e o}=\frac{4 h^{2} \Delta P_{e o}}{\pi^{3} \eta L_{e o}} \sum_{n, o d d}^{\infty} \frac{1}{n^{3}}\left[1-\frac{1}{\cosh \left(n \pi w h^{-1}\right)}\right] \sin (0.5 n \pi) \text {. }
$$

For the given channel geometry $(h=50 \mu \mathrm{m}$ and $w$ $=100 \mu \mathrm{m}$ ) and inserting $\Delta P_{t o t}$, we simplify (A2):

$$
v_{e o}=\frac{2.85 \times 10^{-10}}{\eta L_{e o}} \frac{\Delta P_{t o t}}{2.47} .
$$

The total pressure drop $\Delta P_{\text {tot }}$ is at equilibrium with the pump pressure $\Delta P_{p}$ during velocity measurements. Inserting Eq. (9) into Eq. (A3) gives the specific geometry coefficient $\varphi$ for the heated pump:

$$
\phi=\frac{2.85 \times 10^{-10}}{2.47 L_{e o}} .
$$

Figure 12 shows theoretical velocity-frequency spectra for different $\Delta T$ and fluid conductivities (compare to Fig. 1).

\section{APPENDIX B}

For the numerical 2D model, we employed the COMSOL MULTIPHYSICS modules for electrostatics, general heat transfer, conductive media dc, and laminar fluid flow in combination with Eq. (1).

\section{Module: Electrostatics (with small in-plane currents)}

The Poisson equation $-\nabla\left[\left(\sigma+j \varpi \varepsilon_{0} \varepsilon_{r}\right) \nabla V\right]=0$ was applied in this subdomain. No current flow was allowed across insulating outside boundaries $(n \cdot J=0$, with $n$ the normal unit vector, $J$ the current density). Electrode boundary potentials 
were set to zero and $\mathrm{V}_{\text {rms }}$, respectively. Internal boundaries (of two materials with indices 1 and 2) were set to continuity-i.e., $n \cdot\left(J_{1}-J_{2}\right)=0$ - to specify that the normal component of the electric current is continuous.

\section{Module: Conductive media dc}

This module was applied to the heating element. The settings were $-\nabla \cdot \sigma \nabla V=Q_{j}$. The outside boundaries were set to electric insulation $n \cdot J=0$, specifying that a leak of the heating current into the pump medium can be neglected. The electrode boundaries were set to zero and $\mathrm{V}_{\mathrm{dc}}$, respectively. All other interior boundaries were set to continuity $n \cdot\left(J_{1}\right.$ $\left.-J_{2}\right)=0$, specifying that the normal components of the electric current are continuous across internal boundaries.

\section{Module: General heat transfer (with added out-of-plane heat transfer)}

Outside boundaries were set to the external temperature $T_{e x t}$. The subdomain equation is $\nabla \cdot\left(-d_{z} k \nabla T\right)=d_{z} Q-d_{z} \rho C_{p} v \cdot \nabla T+h_{u}\left(T_{e x t, u}-T\right)+h_{d}\left(T_{e x t, d}-T\right)$.

\section{Module: Incompressible Navier-Stokes media}

The subdomain equations are: $\rho(v \cdot \nabla) v=\nabla \cdot[-p I$ $\left.+\eta\left(\nabla v+(\nabla v)^{T}\right)\right]+F$ and $\nabla \cdot v=0$. Channel boundaries were set to no-slip conditions (velocity $v=0$ at the boundaries).

\section{Variables}

The variables are given in Table II.
[1] D. R. Reyes, D. Iossifidis, P. A. Auroux, and A. Manz, Anal. Chem. 74, 2623 (2002).

[2] P. A. Auroux, D. Iossifidis, D. R. Reyes, and A. Manz, Anal. Chem. 74, 2637 (2002).

[3] W. H. Baumann, M. Lehmann, A. Schwinde, R. Ehret, M. Brischwein, and B. Wolf, Sens. Actuators B 55, 77 (1999).

[4] A. Stett, U. Egert, E. Guenther, F. Hofmann, T. Meyer, W. Nisch, and H. Haemmerle, Anal. Bioanal. Chem. 377, 486 (2003).

[5] G. Krause, S. Lehmann, M. Lehmann, I. Freund, E. Schreiber, and W. Baumann, Biosens. Bioelectron. 21, 1272 (2006).

[6] A. Manz, D. J. Harrison, E. M. J. Verpoorte, J. C. Fettinger, A. Paulus, H. Ludi, and H. M. Widmer, J. Chromatogr. 593, 253 (1992).

[7] M. A. Burns, B. N. Johnson, S. N. Brahmasandra, K. Handique, J. R. Webster, M. Krishnan, T. S. Sammarco, P. M. Man, D. Jones, D. Heldsinger, C. H. Mastrangelo, and D. T. Burke, Science 282, 484 (1998).

[8] U. Bilitewski, M. Genrich, S. Kadow, and G. Mersal, Anal. Bioanal. Chem. 377, 556 (2003).

[9] H. Stone, A. Stroock, and A. Ajdari, Annu. Rev. Fluid Mech. 36, 381 (2004).

[10] D. J. Laser and J. G. Santiago, J. Micromech. Microeng. 14, R35 (2004).

[11] J. S. Jang and S. S. Lee, Sens. Actuators, A 80, 84 (2000).

[12] A. V. Lemoff and A. P. Lee, Sens. Actuators B 63, 178 (2000).

[13] A. Ajdari, Phys. Rev. E 61, R45 (2000).

[14] T. E. McKnight, C. T. Culbertson, S. C. Jacobson, and J. M. Ramsey, Anal. Chem. 73, 4045 (2001).

[15] S. L. Zeng, C. H. Chen, J. G. Santiago, J. R. Chen, R. N. Zare, J. A. Tripp, F. Svec, and J. M. J. Frechet, Sens. Actuators B 82, 209 (2002).

[16] J. Darabi, M. M. Ohadi, and D. DeVoe, J. Microelectromech. Syst. 10, 98 (2001).

[17] J. Darabi, M. Rada, M. Ohadi, and J. Lawler, J. Microelectro- mech. Syst. 11, 684 (2002).

[18] G. Fuhr, T. Schnelle, and B. Wagner, J. Micromech. Microeng. 4, 217 (1994).

[19] A. Richter, A. Plettner, K. A. Hofmann, and H. Sandmaier, Sens. Actuators, A 29, 159 (1991).

[20] A. B. D. Brown, C. G. Smith, and A. R. Rennie, Phys. Rev. E 63, 016305 (2000).

[21] A. Ramos, A. Gonzalez, A. Castellanos, N. G. Green, and H. Morgan, Phys. Rev. E 67, 056302 (2003).

[22] A. Ramos, H. Morgan, N. G. Green, A. Gonzalez, and A. Castellanos, J. Appl. Phys. 97, 084906 (2005).

[23] J. Gimsa, G. Fuhr, R. Glaser, and R. Hagedorn, GDR Patent No. DD 279984A1 (1990).

[24] R. Hagedorn, G. Fuhr, T. Muller, and J. Gimsa, Electrophoresis 13, 49 (1992).

[25] N. G. Green, A. Ramos, A. Gonzalez, A. Castellanos, and H. Morgan, J. Electrost. 53, 71 (2001).

[26] J. Gimsa and M. Holtappels, International patent application No. WO/2005/001286 (2005).

[27] M. Holtappels, J. Gimsa, and M. Stubbe, International patent application No. WO/2007/098910 (2007).

[28] M. Stubbe, M. Holtappels, and J. Gimsa, J. Phys. D 40, 6850 (2007).

[29] A. Ramos, H. Morgan, N. G. Green, and A. Castellanos, J. Phys. D 31, 2338 (1998).

[30] D. R. Lide, Handbook of Chemistry and Physics (CRC Press, Boca Raton, 1998).

[31] N. G. Green, A. Ramos, A. Gonzalez, A. Castellanos, and H. Morgan, J. Phys. D 33, L13 (2000).

[32] J. Gimsa, T. Müller, T. Schnelle, and G. Fuhr, Biophys. J. 71, 495 (1996).

[33] H. Bruus, Theoretical Microfluidics (Oxford University Press, Oxford, 2007).

[34] www.cosmol.com 